\title{
Positive Solutions of Three-Order Delayed Periodic Boundary Value Problems
}

\author{
Na Wang \\ Department of Applied Mathematics, Shanghai Institute of Technology, Shanghai 201418, China \\ Correspondence should be addressed to Na Wang; wangna1621@yeah.net
}

Received 26 June 2017; Accepted 11 September 2017; Published 4 October 2017

Academic Editor: Zhengqiu Zhang

Copyright (C) $2017 \mathrm{Na}$ Wang. This is an open access article distributed under the Creative Commons Attribution License, which permits unrestricted use, distribution, and reproduction in any medium, provided the original work is properly cited.

Our main purpose is to consider the existence of positive solutions for three-order two-point boundary value problem in the following form: $u^{\prime \prime \prime}(t)+\rho^{3} u(t)=f(t, u(t-\tau)), 0 \leq t \leq 2 \pi, u^{(i)}(0)=u^{(i)}(2 \pi), i=1,2, u(t)=\sigma,-\tau \leq t \leq 0$, where $\sigma, \rho$, and $\tau$ are given constants satisfying $\tau \in(0, \pi / 2)$. Some inequality conditions on $\rho^{3} u-f(t, u)$ guaranteeing the existence and nonexistence of positive solutions are presented. Our discussion is based on the fixed point theorem in cones.

\section{Introduction and Preliminaries}

We consider the existence of positive solutions for the following two-point BVP:

$$
\begin{aligned}
u^{\prime \prime \prime}(t)+\rho^{3} u(t) & =f(t, u(t-\tau)), \quad 0 \leq t \leq 2 \pi \\
u^{(i)}(0) & =u^{(i)}(2 \pi), \quad i=1,2, \\
u(t) & =\sigma, \quad-\tau \leq t \leq 0,
\end{aligned}
$$

where $\rho^{3} u-f(t, u) \in C([0,2 \pi],[0,+\infty)), \rho^{3} u-f(t, u) \geq 0$. $\sigma, \rho$, and $\tau$ are given constants satisfying $\tau \in(0, \pi / 2)$.

BVPs play an important role in many branches of mathematics, physics, and engineering and have been a focus in tens of years. They have special importance in the theory and applications, and significant progress has been made on the existence, multiplicity, and nonexistence of positive solutions. Many methods and theorems based on the fixed point theory on cone, the upper and lower solution, Mawhin's coincidence degree, and variational method and so on have been employed commonly in recent years to show the existence of positive or multiple positive solutions [1-9].
For example, Chu and Zhou [3] studied the boundary value problem:

$$
\begin{aligned}
u^{\prime \prime \prime}(t)+\rho^{3} u(t) & =f(t, u(t)), \quad 0 \leq t \leq 2 \pi \\
u^{(i)}(0) & =u^{(i)}(2 \pi), \quad i=0,1,2 .
\end{aligned}
$$

Here, $\rho \in(0,1 / \sqrt{3})$ is a positive constant and the nonlinearity $f(t, u)$ may be singular at $u=0$. The proof relies on a nonlinear alternative of Leray-Schauder type and on Krasnoselskii fixed point theorem on compression and expansion of cones. In [5], Cabada investigated the solvability of two-point BVP:

$$
\begin{aligned}
L_{n} u(t) & =f(t, u(t)), \\
u^{(i)}(0)-u^{(i)}(2 \pi) & =\mu_{i} \in R,
\end{aligned}
$$

where $L_{n}$ is an $n$ th-order linear operator and $f$ is a Carathéodory function. By using the method of lower and upper solutions, the existence results are obtained.

As far as the author knows, however, there are no results which contain the existence criteria of positive solutions to problem (1) with delay. Since the time delays in some equations with practical application background are often very small, they are easier to miss. As we know, even a small delay is also likely to have an important impact on the stability 
of the system. Therefore it is necessary for us to consider the influence of delays. Our work is based on such a background. Motivated by this fact, we will study mainly this problem here. By using fixed point theorem, we obtain some new results. The interest is that the result (Theorem 2) is related to the deviating argument $\tau$. Meanwhile, we give an example to demonstrate our result.

Our main results will hinge on an application of the Leggett-Williams fixed point theorem. For the convenience of the reader, we include here the necessary definitions from cone theory in Banach space.

Throughout this paper, the sign [] stands for deviation operation; namely, $[u] \equiv u(t-\tau)$. Let $0<d<a$ be given and suppose that $f$ satisfies

$$
\begin{aligned}
& \left(H_{1}\right) \lim _{u \rightarrow+\infty}\left(\left(\rho^{3} u-f(t,[u])\right) / u\right)<1 / 4 \pi^{3} ; \\
& \left(H_{2}\right) \rho^{3} u-f(t,[u])<d / 4 \pi^{3} \text { for all } 0 \leq u \leq d ; \\
& \left(H_{3}\right) \rho^{3} u-f(t,[u])>96 a / \pi \text { for all } a \leq u \leq 8 a \\
& \left(H_{2}^{\prime}\right) \lim _{u \rightarrow 0}\left(\left(\rho^{3} u-f(t,[u])\right) / u\right)<1 / 4 \pi^{3}
\end{aligned}
$$

Green's function for BVP (1) is

$$
G(t, s)= \begin{cases}\frac{s(s-t)(2 \pi-t)}{4 \pi}, & 0 \leq s \leq t \leq 2 \pi \\ \frac{t(t-s)(2 \pi-s)}{4 \pi}, & 0 \leq t \leq s \leq 2 \pi\end{cases}
$$

Suppose that $u(t)$ is a solution of BVP (1); we have

$$
\begin{aligned}
u(t) & =\int_{0}^{2 \pi} G(t, s)\left[f(s, u(s-\tau))-\rho^{3} u(t)\right] d s \\
& =\int_{0}^{t} \frac{s(t-s)(2 \pi-t)}{4 \pi}\left[\rho^{3} u(t)\right. \\
& -f(s, u(s-\tau))] d s \\
& +\int_{t}^{2 \pi} \frac{t(s-t)(2 \pi-s)}{4 \pi}\left[\rho^{3} u(t)\right. \\
& -f(s, u(s-\tau))] d s \geq \int_{0}^{t} \frac{s(2 \pi-s)^{2}}{16 \pi^{3}}\left[\rho^{3} u(t)\right. \\
& -f(s, u(s-\tau))] d s+\int_{t}^{2 \pi} \frac{s(2 \pi-s)^{2}}{16 \pi^{3}}\left[\rho^{3} u(t)\right. \\
& -f(s, u(s-\tau))] d s=\frac{1}{16 \pi^{3}} \int_{0}^{2 \pi} s(2 \pi-s)^{2} \\
& \cdot\left[\rho^{3} u(t)-f(s, u(s-\tau))\right] d s .
\end{aligned}
$$

Since $\rho^{3} u-f(t,[u]) \geq 0$, there exists an interval $J=$ $\left[\delta_{1}, \delta_{2}\right] \subset(0,2 \pi)$ such that $\min _{t \in\left[\delta_{1}, \delta_{2}\right]}\left(\rho^{3} u-f(t,[u])\right)>0$. Thus $u(t)>0$.

Next we state the Leggett-Williams fixed point theorem.
Lemma 1 (see [6]). Let $\Phi: \overline{P_{c}} \rightarrow \overline{P_{c}}$ be a completely continuous operator and let $\eta$ be a nonnegative continuous concave functional on $P$ such that $\eta(u) \leq\|u\|$ for all $u \in \overline{P_{c}}$. Suppose that there exist $0<d<a<b \leq c$ such that
(a) $\{u \in P(\eta, a, b) \mid \eta(u)>a\} \neq \emptyset$ and $\eta(\Phi u)>a$ for $u \in P(\eta, a, b)$,
(b) $\|\Phi u\|<d$ for $\|u\| \leq d$,
(c) $\eta(\Phi u)>a$ for $u \in P(\eta, a, c)$ with $\|\Phi u\|>b$.

Then $\Phi$ has at least three fixed points $u_{1}, u_{2}$, and $u_{3}$ such that $\left\|u_{1}\right\|<d, a<\eta\left(u_{2}\right)$ and $\left\|u_{3}\right\|>d$ with $\eta\left(u_{3}\right)<a$.

\section{Main Result}

Theorem 2. Suppose that conditions $\left(H_{1}\right),\left(H_{2}\right)$, and $\left(H_{3}\right)$ hold. Then BVP (1) has at least three positive solutions $y_{1}, y_{2}$, and $y_{3}$ such that $\left\|y_{1}\right\|<a, \eta\left(y_{2}\right)>d,\left\|y_{3}\right\|>a$, and $\eta\left(y_{3}\right)<a$.

Proof. Let $\mathscr{Z}$ denote the Banach space $C[0,2 \pi]$ with the norm

$$
\|u\|_{[0,2 \pi]}=\sup \{|u(t)|: 0 \leq t \leq 2 \pi\}
$$

and define the cone $P \subset \mathscr{Z}$ by

$$
P=\{u \in \mathscr{Z}: u(t) \geq 0\} .
$$

Let $\eta: P \rightarrow[0,+\infty)$ be the nonnegative continuous concave functional

$$
\eta(u)=\min _{\pi / 4 \leq t \leq \pi / 2} u(t), \quad \forall u \in P
$$

and let $\Phi: P \rightarrow P$ be the operator

$$
(\Phi u) t=\int_{0}^{2 \pi} G(t, s)\left[f(s, u(s-\tau))-\rho^{3} u(s)\right] d s
$$

It is easy to see that $\Phi: \overline{P_{8 a}} \rightarrow \bar{P}(b=8 a)$ is completely continuous and $u(t) \equiv(5 a / 2)(>a)$ is an element of $P(\eta, a, 8 a)$. Since $\eta(u)=\eta(5 a / 2)>a$, we have $\{u \mid u \in$ $P(\eta, a, 8 a), \eta(u)>a\} \neq \emptyset$.

Choose $u \in P(\eta, a, 8 a)$ and $\tau \in(0, \pi / 4) \cup(\pi / 4, \pi / 2)$; then

$$
\begin{aligned}
& \eta(\Phi u)=\min _{\pi / 4 \leq t \leq \pi / 2} \int_{0}^{2 \pi} G(t, s)[f(s, u(s-\tau)) \\
& \left.-\rho^{3} u(s)\right] d s \\
& \geq \min _{\pi / 4 \leq t \leq \pi / 2} \int_{0}^{2 \pi} \frac{s(2 \pi-s)^{2}}{16 \pi^{3}}\left[\rho^{3} u(s)\right. \\
& -f(s, u(s-\tau))] d s \geq \frac{6 a}{\pi^{4}} \int_{\pi / 4+\tau}^{\pi / 2+\tau} s(2 \pi-s)^{2} d s \\
& \quad=\left.\frac{6 a}{\pi^{4}}\left[2 \pi^{2} s^{2}-\frac{4}{3} \pi s^{3}+\frac{1}{4} s^{4}\right]\right|_{\pi / 4+\tau} ^{\pi / 2+\tau}>\frac{6 a}{\pi^{4}}\left(\frac{17}{72} \pi^{4}\right. \\
& \left.+\frac{23}{64} \pi^{3} \tau+\frac{\pi}{4} \tau^{3}-\frac{23}{32} \pi^{2} \tau^{2}\right)>a
\end{aligned}
$$


From $\left(H_{1}\right)$, there exist two positive constants $\lambda$ and $M$ with $0<\lambda<1 / 4 \pi^{3}$ such that

$$
\rho^{3} u-f(t,[u]) \leq \lambda u, \quad u>M
$$

If we take $L=\max _{0 \leq u \leq M}\left(\rho^{3} u-f(t,[u])\right)$, then

$$
0 \leq \rho^{3} u-f(t,[u]) \leq \lambda u+L, \quad 0 \leq u \leq+\infty
$$

Let $r>\max \left\{4 \pi^{3} L /\left(1-4 \pi^{3} \lambda\right), 8 a\right\}$ and $u \in \overline{P_{r}}(b=8 a, c=r)$; from (10) we get

$\|\Phi u\|$

$$
\begin{aligned}
& =\max _{0 \leq t \leq 2 \pi} \int_{0}^{2 \pi} G(t, s)\left[f(s, u(s-\tau))-\rho^{3} u(s)\right] d s \\
& \leq 4 \pi^{3}(\lambda u+L) \leq 4 \pi^{3}(\lambda r+L)<r .
\end{aligned}
$$

So $\Phi u \in P_{r}$ and $\Phi: \overline{P_{r}} \rightarrow \overline{P_{r}}$ is completely continuous. From (8), condition (a) of Leggett-Williams theorem is satisfied.

Choose $u \in \overline{P_{d}}$; then

$$
\begin{aligned}
& \|\Phi u\|=\max _{0 \leq t \leq 2 \pi} \int_{0}^{2 \pi} G(t, s) \\
& \cdot\left[f(s, u(s-\tau))-\rho^{3} u(s)\right] d s<\frac{d}{4 \pi^{3}} \\
& \cdot \max _{0 \leq t \leq 2 \pi}\left(\int_{0}^{t} \frac{s(t-s)(2 \pi-t)}{4 \pi} d s\right. \\
& \left.+\int_{t}^{2 \pi} \frac{t(s-t)(2 \pi-s)}{4 \pi} d s\right)<\frac{d}{4 \pi^{3}}\left(\int_{0}^{t} \frac{8 \pi^{3}}{4 \pi} d s\right. \\
& \left.+\int_{t}^{2 \pi} \frac{8 \pi^{3}}{4 \pi} d s\right)=d .
\end{aligned}
$$

Therefore, condition (b) is satisfied.

Choose $y \in \overline{P_{8 a}}$ and $\Phi y \neq 0$; then

$$
\begin{aligned}
& \eta(\Phi u)=\min _{\pi / 4 \leq t \leq \pi / 2} \int_{0}^{2 \pi} G(t, s)[f(s, u(s-\tau)) \\
& \left.-\rho^{3} u(s)\right] d s \\
& \quad=\min _{\pi / 4 \leq t \leq \pi / 2}\left(\int _ { 0 } ^ { t } \frac { s ( t - s ) ( 2 \pi - t ) } { 4 \pi } \left[\rho^{3} u(s)\right.\right. \\
& -f(s, u(s-\tau))] d s \\
& +\int_{t}^{2 \pi} \frac{t(s-t)(2 \pi-s)}{4 \pi}\left[\rho^{3} u(s)\right. \\
& -f(s, u(s-\tau))] d s)=\min _{\pi / 4 \leq t \leq \pi / 2}\left(\int_{0}^{t} \frac{2 \pi-t}{2 \pi-s}\right.
\end{aligned}
$$

$$
\begin{aligned}
& \cdot \frac{s|t-s|(2 \pi-t)}{4 \pi}\left[\rho^{3} u(s)-f(s, u(s-\tau))\right] d s \\
& +\int_{t}^{2 \pi} \frac{t}{s} \cdot \frac{s|s-t|(2 \pi-s)}{4 \pi}\left[\rho^{3} u(s)\right. \\
& -f(s, u(s-\tau))] d s)>\frac{2 \pi-\pi / 2}{2 \pi} \\
& \cdot \int_{0}^{t} \frac{s|t-s|(2 \pi-t)}{4 \pi}\left[\rho^{3} u(s)\right. \\
& -f(s, u(s-\tau))] d s+\frac{\pi / 4}{2 \pi} \\
& \cdot \int_{t}^{2 \pi} \frac{s|s-t|(2 \pi-s)}{4 \pi}\left[\rho^{3} u(s)\right. \\
& -f(s, u(s-\tau))] d s>\frac{1}{8}\|\Phi u\| .
\end{aligned}
$$

So, if $\|\Phi u\|>8 a$, then $\eta(\Phi u)>(1 / 8)\|\Phi u\|>a$, which implies that condition (c) is also satisfied. An application of LeggettWilliams fixed point theorem yields the result.

Theorem 3. Suppose that conditions $\left(H_{1}\right),\left(H_{2}^{\prime}\right)$, and $\left(H_{3}\right)$ hold; then BVP (1) has at least three positive solutions $y_{1}, y_{2}$, and $y_{3}$ such that $\left\|y_{1}\right\|<a, \eta\left(y_{2}\right)>d,\left\|y_{3}\right\|>a$, and $\eta\left(y_{3}\right)<a$.

The proof of Theorem 3 is similar to that of Theorem 2 and is hence omitted.

Remark 4. The extensions to Theorem 2 can be obtained by Theorem 5 , where $1 / 4 \pi^{3}$ of $\left(H_{1}\right)$ and $\left(H_{2}\right)$ is replaced by $\delta$ and $\gamma$, respectively. We omit the detail.

Theorem 5. Suppose that conditions

$$
\begin{aligned}
& \left(H_{1}^{*}\right) \lim _{u \rightarrow+\infty}\left(\left(\rho^{3} u-f(t,[u])\right) / u\right)<\delta, \\
& \left(H_{2}^{*}\right) \lim _{u \rightarrow 0}\left(\left(\rho^{3} u-f(t,[u])\right) / u\right)<\gamma, \\
& \text { and }\left(H_{3}\right) \text { hold. }
\end{aligned}
$$

Then BVP (1) has at least three positive solutions; here $\delta, \gamma<$ $1 / 4 \pi^{3}$.

Now we present a result on the nonexistence of positive solutions of BVP (1).

Theorem 6. BVP (1) has no positive solutions if

(i) $\lim _{u \rightarrow+\infty} \inf _{t \in[0,2 \pi]}\left(\left(\rho^{3} u-f(t,[u])\right) /\|u\|\right)>1 / 4 \pi^{3}$;

(ii) $\lim _{u \rightarrow+\infty} \sup _{t \in[0,2 \pi]}\left(\left(\rho^{3} u-f(t,[u])\right) /\|u\|\right)<1 / 4 \pi^{3}$;

(iii) $\left(\rho^{3} u-f(t,[u])\right) /\|u\|>1 / 4 \pi^{3}$; or

(iv) $\left(\rho^{3} u-f(t,[u])\right) /\|u\|<1 / 4 \pi^{3}$,

where $\|u\|$ is defined by $(*)$.

Proof. (a) From condition (i), there exist two positive constants $\lambda_{3}$ and $M_{3}$ with $0<\lambda_{3}<1 / 4 \pi^{3}$ such that

$$
\rho^{3} u(t)-f(t,[u])>\lambda_{3}\|u\|, \quad u>M_{3} .
$$


If we take $L_{1}^{*}=\min _{0 \leq u \leq M_{3}}\left(\rho^{3} u(t)-f(t,[u])\right)$, then

$$
\rho^{3} u(t)-f(t,[u])>\lambda_{3}\|u\|+L_{1}^{*}, \quad 0 \leq u \leq+\infty .
$$

Assume that BVP (1) has a positive solution $u$ with $\|u\|=r$. Then $u$ is a fixed point of the map $\Phi$ defined by $(* *)$ and hence

$$
\begin{aligned}
r & =\|\Phi u\| \\
& =\max _{0 \leq t \leq 2 \pi} \int_{0}^{2 \pi} G(t, s)\left[f(s, u(s-\tau))-\rho^{3} u(t)\right] d s \\
& >4 \pi^{3}\left(\lambda_{3}\|u\|+L_{1}^{*}\right)=4 \pi^{3}\left(\lambda_{3} r+L_{1}^{*}\right)>r,
\end{aligned}
$$

which is a contradiction.

(b) From condition (ii), there exist two positive constants $\lambda_{4}$ and $M_{4}$ with $0<\lambda_{4}<1 / 4 \pi^{3}$ such that

$$
\rho^{3} u(t)-f(t,[u])<\lambda_{4}\|u\|, \quad u>M_{4} .
$$

If we take $L_{2}^{*}=\min _{0 \leq u \leq M_{3}}\left(\rho^{3} u(t)-f(t,[u])\right)$, then

$$
\rho^{3} u(t)-f(t,[u])<\lambda_{4}\|u\|+L_{2}^{*}, \quad 0 \leq u \leq+\infty .
$$

Assume that BVP (1) has a positive solution $u$ with $\|u\|=r$. Then $u$ is a fixed point of the map $\Phi$ defined by $(* *)$ and hence

$$
\begin{aligned}
r & =\|\Phi u\| \\
& =\max _{0 \leq t \leq 2 \pi} \int_{0}^{2 \pi} G(t, s)\left[f(s, u(s-\tau))-\rho^{3} u(t)\right] d s \\
& >4 \pi^{3}\left(\lambda_{4}\|u\|+L_{2}^{*}\right)=4 \pi^{3}\left(\lambda_{4} r+L_{2}^{*}\right)<r,
\end{aligned}
$$

which is a contradiction.

(c) Assume that BVP (1) has a positive solution $u$ with $\|u\|=r$. Then $u$ is a fixed point of the map $\Phi$ and hence

$$
\begin{aligned}
r & =\|\Phi u\| \\
& =\max _{0 \leq t \leq 2 \pi} \int_{0}^{2 \pi} G(t, s)\left[f(s, u(s-\tau))-\rho^{3} u(t)\right] d s \\
& >4 \pi^{3} \cdot \frac{1}{4 \pi^{3}}\|u\|=r
\end{aligned}
$$

which is a contradiction.

(d) The proof of case (iii) is similar to that of case (iv) and is hence omitted.

Remark 7. The extensions to Theorem 6 can be obtained by Theorem 8 , where $1 / 4 \pi^{3}$ of (i)-(iv) is replaced by $\alpha, \beta, \theta$, and $\nu$, respectively. Here $\alpha, \beta, \theta, v<1 / 4 \pi^{3}$. We omit the detail.

Theorem 8. BVP (1) has no positive solutions if

(i) $\lim _{u \rightarrow+\infty} \inf _{t \in[0,2 \pi]}\left(\left(\rho^{3} u-f(t,[u])\right) /\|u\|\right)>\alpha$;

(ii) $\lim _{u \rightarrow+\infty} \sup _{t \in[0,2 \pi]}\left(\left(\rho^{3} u-f(t,[u])\right) /\|u\|\right)<\beta$;

(iii) $\left(\rho^{3} u-f(t,[u])\right) /\|u\|>\theta$; or

(iv) $\left(\rho^{3} u-f(t,[u])\right) /\|u\|<v$,

where $\|u\|$ is defined by $(*)$.
Example 9. Let us consider the following equation:

$$
\begin{aligned}
u^{\prime \prime \prime}(t)+\rho^{3} u(t) & =f\left(t, u\left(t-\frac{\pi}{8}\right)\right), \quad 0 \leq t \leq 2 \pi \\
u^{(i)}(0) & =u^{(i)}(2 \pi), \quad i=1,2, \\
u(0) & =0, \quad-\frac{\pi}{8} \leq t \leq 0,
\end{aligned}
$$

where

$$
\begin{aligned}
& \rho^{3} u(t)-f\left(t, u\left(t-\frac{\pi}{8}\right)\right) \\
& = \begin{cases}\frac{e^{u(t-\pi / 8)}}{32 \pi^{3}}, & 0 \leq t \leq 2 \pi, 0 \leq u \leq \frac{1}{2} ; \\
6 u\left(t-\frac{\pi}{8}\right)-3, & 0 \leq t \leq 2 \pi, \frac{1}{2} \leq u \leq 1 ; \\
\pi^{2}\left[e^{u(t-\pi / 8)}+u^{2}(t)\right]+17, & 0 \leq t \leq 2 \pi, 1 \leq u \leq 8 ; \\
\frac{u(t-\pi / 8)}{5 \pi^{3}} & 0 \leq t \leq 2 \pi, u>8 .\end{cases}
\end{aligned}
$$

Let $d=1 / 2, a=1, c=8$, and $r>8$; then we have $\rho^{3} u(t)-f(t, u(t-\pi / 8)) \in C([0,2 \pi],[0,+\infty)), \rho^{3} u-f(t,[u]) \geq$ 0 , and $\rho^{3} u(t)-f(t, u(t-\pi / 8))<1 / 8 \pi^{3}$ for all $0 \leq u \leq 1 / 2$, $\rho^{3} u(t)-f(t, u(t-\pi / 8))>96 / \pi$ for all $1 \leq u \leq 8$, and $\lim _{u \rightarrow+\infty}\left(\left(\rho^{3} u-f(t,[u])\right) / u\right)<1 / 4 \pi^{3}$. One can easily see that assumptions $\left(H_{1}\right),\left(H_{2}\right)$, and $\left(H_{3}\right)$ hold. So by applying Theorem 2, BVP (21) has at least three positive solutions $u_{1}, u_{2}$, and $u_{3}$ and satisfied $\left\|u_{1}\right\|<1 / 2, \eta\left(u_{2}\right)>8,\left\|u_{3}\right\|>1 / 2$, and $\eta\left(u_{3}\right)<8$.

Example 10. Let us consider the following equation:

$$
\begin{aligned}
u^{\prime \prime \prime}(t)+\rho^{3} u(t) & =f(t, u(t-\tau)), \quad 0 \leq t \leq 2 \pi \\
u^{(i)}(0) & =u^{(i)}(2 \pi), \quad i=1,2, \\
u(0) & =1, \quad-\frac{\pi}{3} \leq t \leq 0 .
\end{aligned}
$$

(1) Let $\rho^{3} u(t)-f(t, u(t-\tau))=4 u(t)+u(t-\pi / 3)$; it is clear that

$$
\lim _{u \rightarrow+\infty} \inf _{t \in[0,2 \pi]}\left(\frac{\rho^{3} u-f(t,[u])}{\|u\|}\right)>\frac{1}{4 \pi^{3}} .
$$

Therefore, by applying Theorem 6(i), BVP (23) has no positive solutions.

(2) Let $\rho^{3} u(t)-f(t, u(t-\tau))=\left(1 / 8 \pi^{3}\right)(u(t)+u(t-\pi / 3))$; it is clear that

$$
\lim _{u \rightarrow+\infty} \sup _{t \in[0,2 \pi]}\left(\frac{\rho^{3} u-f(t,[u])}{\|u\|}\right)<\frac{1}{4 \pi^{3}}
$$

Therefore, by applying Theorem 6(ii), BVP (23) has no positive solutions. 
The numerical results of Example 10 by Mathematica 7.0 are in the following:

$$
\begin{aligned}
\text { In }[18]:= & \mathbf{s o l}=\text { NDSolve }\left[\left\{\mathbf{x}^{\prime \prime \prime}[\mathbf{t}]==-\mathbf{4} \mathbf{x}[\mathbf{t}]-\mathbf{x}[\mathbf{t}-\mathbf{P i} / \mathbf{3}], \mathbf{x}[\mathbf{t} / ; \mathbf{t} \leq \mathbf{0}]==\mathbf{1}, \mathbf{x}^{\prime}[\mathbf{0}]==\mathbf{x}^{\prime}[\mathbf{2} \boldsymbol{\pi}], \mathbf{x}^{\prime \prime}[\mathbf{0}]==\mathbf{x}^{\prime \prime}[\mathbf{2} \boldsymbol{\pi}]\right\}, \mathbf{x},\{\mathbf{t}, \mathbf{0}, \mathbf{2} \boldsymbol{\pi}\}\right] \\
& \text { Function : : slotn : Slot number } 5 \text { in }\{\# 3, \# 4,-4 \# 2-\# 5\} \text { \& cannot be filled from }(\{\# 3, \# 4,-4 \# 2-\# 5\} \&)[0 ., 0 ., 0 ., 0 .] . \gg
\end{aligned}
$$

NDSolve : : ndnum : Encountered non-numerical value for a derivative at $t==0 \therefore \gg$

$$
\begin{aligned}
& \text { Out [18] = NDSolve }\left[\left\{x^{(3)}[t]==-4 x[t]-x\left[-\frac{\pi}{3}+t\right], x[t / ; t \leq 0]==1, x^{\prime}[0]==x^{\prime}[2 \pi], x^{\prime \prime}[0]==x^{\prime \prime}[2 \pi]\right\}, x,\{t, 0,2 \pi\}\right] \\
& \text { In [19]:= } \mathbf{s o l}=\mathbf{N D S o l v e}\left[\left\{\mathbf{x}^{\prime \prime \prime}[\mathbf{t}]==(-\mathbf{x}[\mathbf{t}]-\mathbf{x}[\mathbf{t}-\mathbf{P i} / \mathbf{3}]) /\left(\mathbf{8} \boldsymbol{\pi}^{3}\right), \mathbf{x}[\mathbf{t} / ; \mathbf{t} \leq \mathbf{0}]==\mathbf{1}, \mathbf{x}^{\prime}[\mathbf{0}]==\mathbf{x}^{\prime}[\mathbf{2 \pi}], \mathbf{x}^{\prime \prime}[\mathbf{0}]==\mathbf{x}^{\prime \prime}[\mathbf{2 \pi}]\right\}, \mathbf{x},\{\mathbf{t}, \mathbf{0}, \mathbf{2 \pi}\}\right] \\
& \text { Function : : slotn : Slot number } 5 \text { in }\left\{\# 3, \# 4, \frac{-\# 2-\# 5}{8 \pi^{3}}\right\} \text { \& cannot be filled from }\left(\left\{\# 3, \# 4, \frac{-\# 2-\# 5}{8 \pi^{3}}\right\} \&\right)[0 ., 0 ., 0 ., 0 .] . \gg
\end{aligned}
$$

NDSolve : : ndnum : Encountered non-numerical value for a derivative at $t==0 \therefore \gg$

$$
\text { Out }[19]=\text { NDSolve }\left[\left\{x^{(3)}[t]==\frac{-x[t]-x[-\pi / 3+t]}{8 \pi^{3}}, x\left[\frac{t}{;} t \leq 0\right]==1, x^{\prime}[0]==x^{\prime}[2 \pi], x^{\prime \prime}[0]==x^{\prime \prime}[2 \pi]\right\}, x,\{t, 0,2 \pi\}\right] \text {. }
$$

Remark 11. From the above example, we see that the result is related to the deviating argument $\tau$, which is different from the Theorem in papers $[1-3,5,6,10]$ and the references therein. The studies indicate that this kind of system with time delays can exhibit triple positive solutions, which shows that three-order two-point boundary value problems have the potential to reproduce the complex dynamics of real applied background in mechanics, engineering, physics, and so on.

Remark 12. The above problem can be extended to the corresponding vector case. For example,

$$
\begin{aligned}
\varepsilon \mathbf{u}^{\prime \prime \prime}(t)+\mu \rho^{3} \mathbf{u}(t) & =\mathbf{f}(t, \mathbf{u}(t-\tau)), \quad 0 \leq t \leq 2 \pi, \\
\mathbf{u}^{(i)}(0) & =\mathbf{u}^{(i)}(2 \pi), \quad i=1,2, \\
\mathbf{u}(t) & =\sigma^{*}, \quad-\tau \leq t \leq 0,
\end{aligned}
$$

where $\mathbf{u}(t) \in C\left(\mathbb{R}, R^{n}\right), \mathbf{f}(t, \mathbf{u}(t-\tau)) \in C\left(R \times R^{n}, R^{n}\right), \sigma^{*} \in R^{n}$, and $\varepsilon$ and $\mu$ are two given parameters. However, if $\varepsilon \ll \mu \rho^{3}$, such equation is a singular perturbation system; it is hard to obtain the positive solution of (27) by using the fixed point theorem.

\section{Disclosure}

The author carried out the main part of this article and the main theorem. The author read and approved the final manuscript.

\section{Conflicts of Interest}

The author declares that there are no conflicts of interest regarding the publication of this paper.

\section{Acknowledgments}

This research was sponsored by the National Science Foundation of China (Grant no. 11401385).

\section{References}

[1] S. Mukhigulashvili, "On a periodic boundary value problem for third order linear functional differential equations," Nonlinear Analysis: Theory, Methods \& Applications, vol. 66, no. 2, pp. 527535, 2007.

[2] L. Kong, S. Wang, and J. Wang, "Positive solution of a singular nonlinear third-order periodic boundary value problem," Journal of Computational and Applied Mathematics, vol. 132, no. 2, pp. 247-253, 2001.

[3] J. Chu and Z. Zhou, "Positive solutions for singular nonlinear third-order periodic boundary value problems," Nonlinear Analysis: Theory, Methods \& Applications, vol. 64, no. 7, pp. 1528-1542, 2006.

[4] Y. Li, "Existence of positive solutions for the cantilever beam equations with fully nonlinear terms," Nonlinear Analysis: Real World Applications, vol. 27, pp. 221-237, 2016.

[5] A. Cabada, "The method of lower and upper solutions for third-order periodic boundary value problems," Journal of Mathematical Analysis and Applications, vol. 195, no. 2, pp. 568589, 1995.

[6] D. J. Guo, "Nonlinear functional analysis," in Jinan, Shandong Science and Technology Press, 2003.

[7] Y. Wu and Z. Zhao, "Positive solutions for third-order boundary value problems with change of signs," Applied Mathematics and Computation, vol. 218, no. 6, pp. 2744-2749, 2011.

[8] N. Wang, J. S. Zhang, S. P. Lu, and Z. . Shen, "A theorem on triple positive solutions for a periodic boundary value problem for a second-order delay differential equation," Journal 
of Mathematical Study. Shuxue Yanjiu, vol. 40, no. 1, pp. 22-28, 2007.

[9] Q. Kong and M. Wang, "Positive solutions of even order system periodic boundary value problems," Nonlinear Analysis: Theory, Methods \& Applications, vol. 72, no. 3-4, pp. 1778-1791, 2010.

[10] A. U. Afuwape, P. Omari, and F. Zanolin, "Nonlinear perturbations of differential operators with nontrivial kernel and applications to third-order periodic boundary value problems," Journal of Mathematical Analysis and Applications, vol. 143, no. 1, pp. 35-56, 1989. 


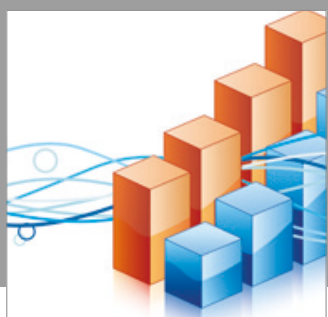

Advances in

Operations Research

vatersals

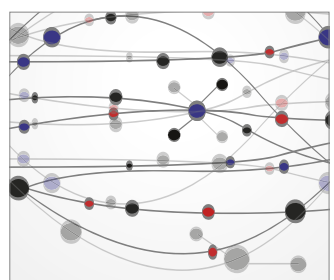

\section{The Scientific} World Journal
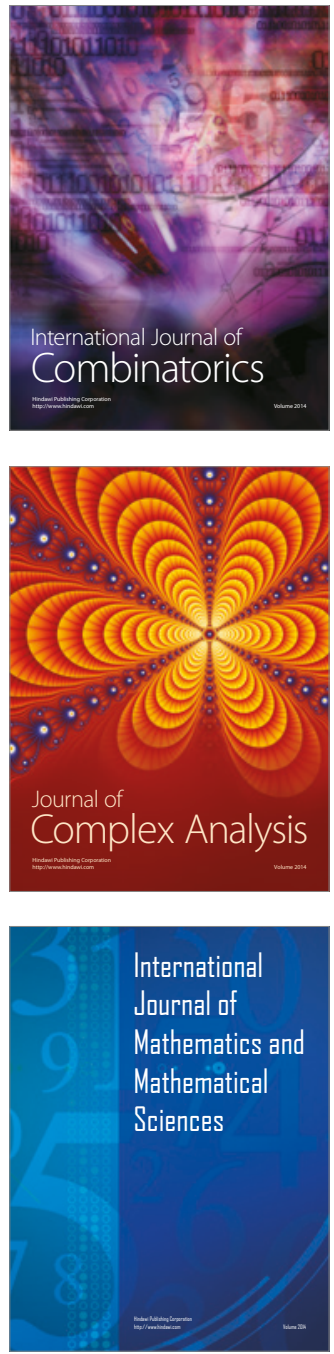
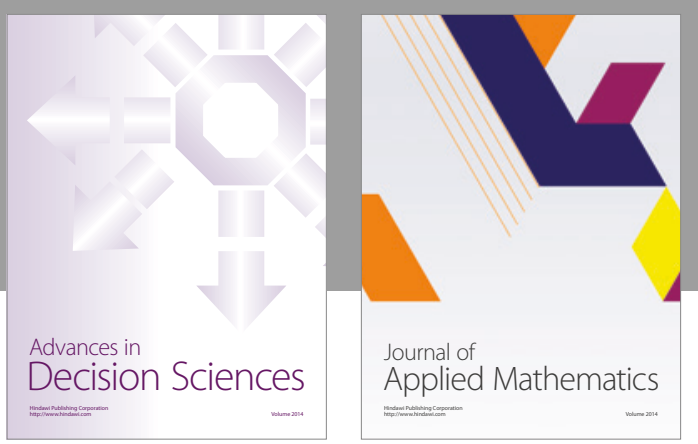

Algebra

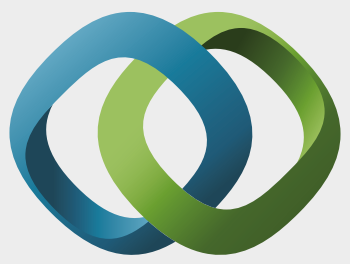

\section{Hindawi}

Submit your manuscripts at

https://www.hindawi.com
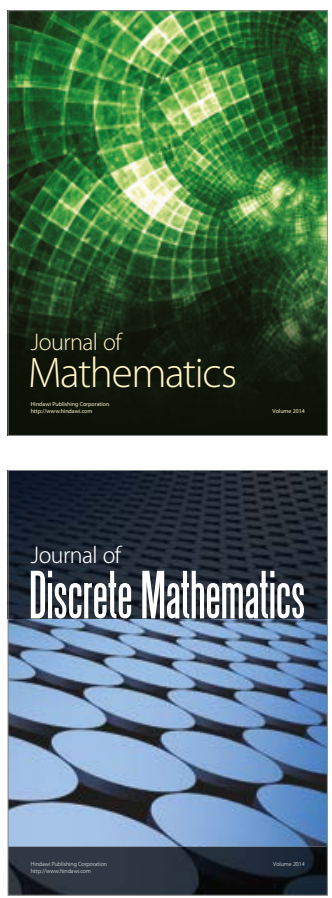

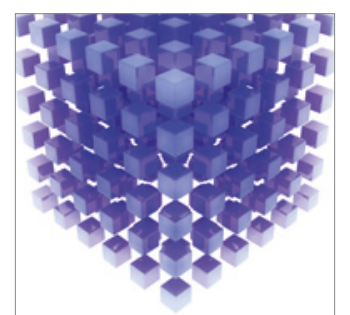

Mathematical Problems in Engineering
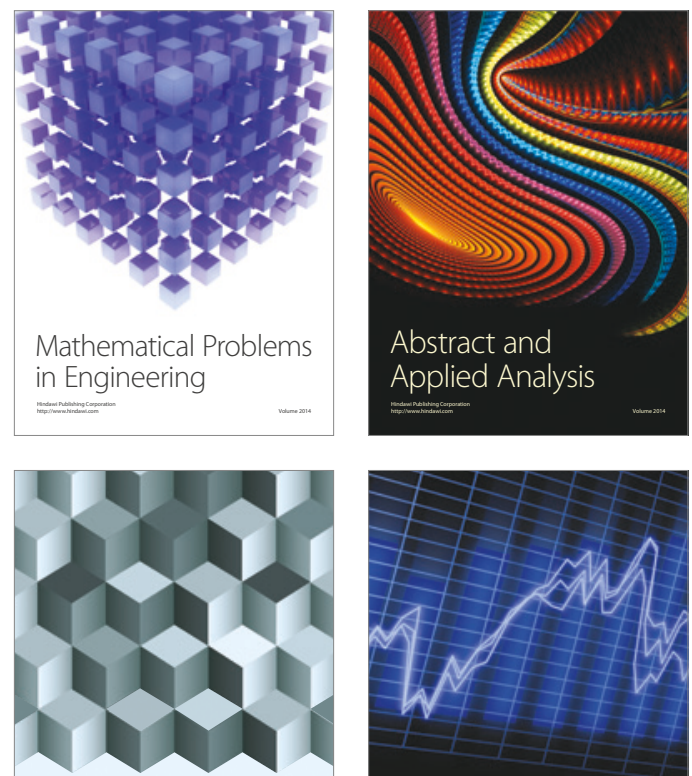

Journal of

Function Spaces

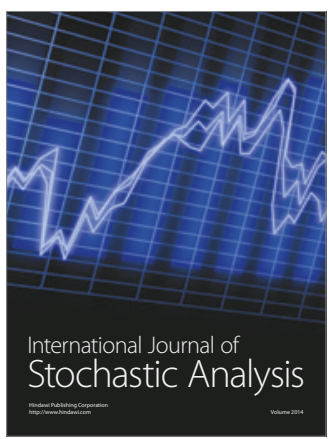

Probability and Statistics
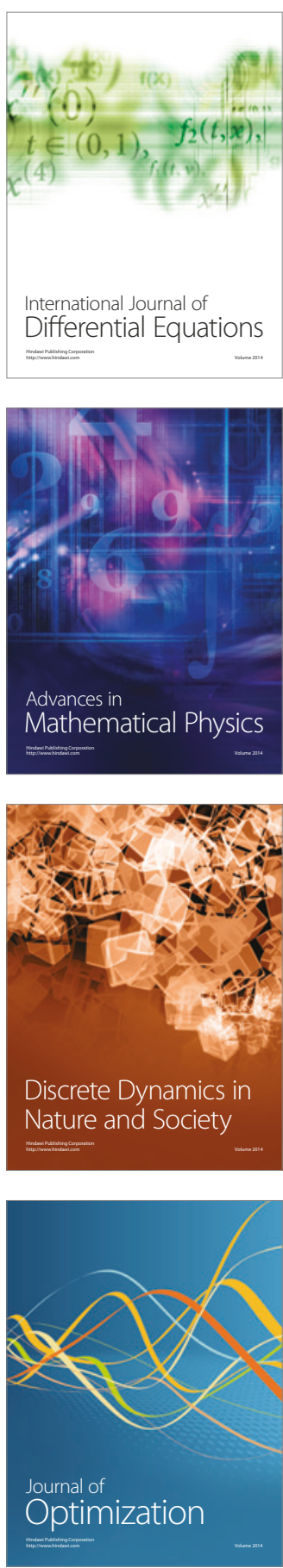